durch die möglichste Unlöslichkeit des Natriumbicarbonates erreicht werden muss. Die Versuche, welche ich in dieser Richtung anstellte, haben auch ein der Theorie entsprechendes Resultat ergeben; es ist mir gelungen, eine Umsetzung von etwa 80 Proc. des angewendeten Kochsalzes zu erzielen. Hinzu tritt hierbei noch der Vortheil, dass die Carbonisation, welche bei dem gewöhnlichen Verfahren mit zunehmender Ausfällung des Bicarbonates immer langsamer vor sich geht, in einer stets concentrirt bleibenden Lösung viel leichter und schneller sich vollzieht, die Kohlensäure wird besser verwerthet. Ferner gibt jede Fällung eine höhere Ausbeute, da durch die stärkere Concentration eine weit grössere Menge Salz in Lösung geht und von dieser grösseren Menge ein höherer Procentsatz zersetzt wird. Hierdurch werden die Apparate, die Maschinenkraft, wie überhaupt die ganze Anlage besser ausgenutzt, auch sind die $z u$ destillirenden Flüssigkeitsmengen kleiner.

Die Anwendung des Verfahrens ist in der Praxis leicht und ohne grössere Anlagekosten auszuführen. Eine einfache Vorrichtung, welche die leichte Einführung des Salzes in die Carbonisatoren gestattet, findet sich abgebildet im Jahresb. $1886 \mathrm{~S}$. ${ }^{*} 281$.

Über die Versuche, welche ich behufs Feststellung der passendsten Concentration der zu dem geschilderten Verfahren angewendeten Lösungen ausgeführt habe, werde ich später berichten.

Bei meinen weiteren Arbeiten über den Ammoniaksodaprocess ist es mir gelungen, noch ein anderes Verfahren aufzufinden, durch welches es ermöglicht wird, den Salzverlust auf ein Minimum zu beschränken.

Ich hatte unter Anderem auch einen Versuch angestellt, indem ich eine vom Bicarbonat abfiltrirte Lösung mit Salz sättigte und dann Ammoniakgas hineinleitete. Die so hergestellte Flüssigkeit carbonisirte ich zum zweiten Mal, um festzustellen, wie weit durch diese doppelte Behandlung die Umsetzung gebracht werden konnte. Es entstand hierbei, als ungefähr sämmtliches Ammoniak in einfaches Carbonat verwandelt war, ein auffallend grosser Niederschlag von Ammonchlorid. In der abfiltrirten Flüssigkeit, welche vorher eine grössere Menge Salmiak als Kochsalz enthalten hatte, war jetzt das umgekehrte Verhältniss eingetreten. Die Fortführung der Carbonisation in der vom Ammonchlorid getrennten Flüssigkeit ergab dann die Ausfällung von Natriumcarbonat. Weitere Versuche zeigten mir, dass sich auf diese Erscheinung ein Verfahren gründen lässt, welches die stetige
Regenerirung der im Ammoniaksodaprocess abfallenden Salmiak-Kochsalzlaugen gestattet, so dass dieselben stets von Neuem in den Betrieb eingeführt werden können.

Leitet man nämlich Ammoncarbonat in Gasform resp. Ammoniak und Kohlensäure in die vom Bicarbonat abfiltrirten Endlaugen und bringt zugleich festes Kochsalz mit denselben in Berührung, wobei die Temperatur am besten etwas hoch gehalten wird, so scheidet sich nach der Abkühlung Chlorammonium in grosser Menge aus, während Ammoncarbonat und Kochsalz in Lösung bleiben. Die so erhaltene Flüssigkeit ist nach der Trennung vom ausgeschiedenen Salmiak direct wieder zur Carbonisation fertig, wie aus folgendem Beispiel ersichtlich ist.

Die Endlauge von der Carbonisation enthielt:

$$
\begin{aligned}
& 9,4 \text { Proc. } \mathrm{Na} \mathrm{Cl}_{19,8}=\mathrm{NH}_{4} \mathrm{Cl} \text {. }
\end{aligned}
$$

Dieselbe Lauge ergab nach der Behandlung mit $\mathrm{NaCl}$ und $\left(\mathrm{NH}_{4}\right)_{2} \mathrm{CO}_{3}$ :

$$
\begin{aligned}
23,1 & \text { Proc. } \\
5,9- & \mathrm{NaCl} \\
18,5- & \left(\mathrm{NH}_{4}\right)_{2} \mathrm{CO}_{3} .
\end{aligned}
$$

Wie man sieht, ist die Zusammensetzung dieser Lösung derart, dass sie ohne Weiteres in den Process wieder eingeführt werden kann.

Für heute beschränke ich mich hinsichtlich des letzteren Verfahrens (Jahresb. 1886 S. 280; Chemzg. 1886 S. 1585) auf diese kurze Mittheilung; ich werde die Resultate meiner näheren Versuche über die dem Verfahren zu Grunde liegenden Reactionen in einer späteren Abhandlung an dieser Stelle mittheilen.

\section{Zur Bestimmung der Phosphorsäure in Düngemitteln.}

Von

W. Mielcke.

In den Laboratorien der landwirthschaftlichen Versuchsstationen ist das Berechnen des Phosphorsäuregehaltes der Düngemittel aus dem gewogenen Magnesiumpyrophosphat eine ständige und ziemlich zeitraubende Arbeit. Ich fertigte daher in letzter Zeit eine Tabelle dafür an, welche seitdem im Laboratorium der chem. Versuchsstation zu Bonn im Gebrauche, und deren Bekanntgabe vielleicht in weiteren Kreisen erwünscht ist. Zur Berechnung ist der hier übliche Factor 128 für $0,5 \mathrm{~g}$ angewandter Substanz $\mathrm{zu}$ Grunde gelegt. 


\begin{tabular}{|c|c|c|c|c|c|c|c|c|c|c|c|c|c|c|c|c|c|c|c|}
\hline 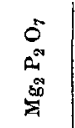 & $\begin{array}{l}5 \\
0 \\
0 \\
0 \\
0 \\
0 \\
0\end{array}$ & 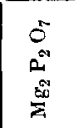 & $\begin{array}{l}0^{\infty} \\
\infty^{\infty}\end{array}$ & $\begin{array}{l}0 \\
0 \\
n^{n} \\
0^{\circ}\end{array}$ & $\begin{array}{l}0^{\infty} \\
0^{\infty}\end{array}$ & 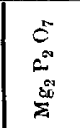 & $\begin{array}{l}0^{n} \\
n^{n}\end{array}$ & 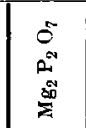 & $\begin{array}{l}0^{10} \\
n^{\infty 1}\end{array}$ & $\begin{array}{l}0 \\
0 \\
0 \\
0 \\
0\end{array}$ & $\begin{array}{l}0^{5} \\
\text { an }\end{array}$ & 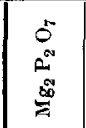 & $\begin{array}{l}0^{\circ} \\
2^{20}\end{array}$ & $\begin{array}{l}5 \\
0 \\
\infty \\
\frac{\omega}{20}\end{array}$ & $\begin{array}{l}0^{\infty} \\
20\end{array}$ & 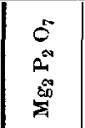 & $\begin{array}{l}0^{\infty} \\
n^{\infty}\end{array}$ & $\begin{array}{l}5 \\
0 \\
A \\
a \\
a \\
a\end{array}$ & $\begin{array}{l}\infty \\
\infty \\
\infty\end{array}$ \\
\hline $\begin{array}{r}400 \\
2 \\
4 \\
6 \\
8\end{array}$ & $\begin{array}{l}\mathbf{5 , 1 2} \\
\mathbf{5 , 1 5} \\
\mathbf{5 . 1 7} \\
\mathbf{5 , 2 0} \\
\mathbf{5 , 2 2}\end{array}$ & $\begin{array}{r}410 \\
2 \\
4 \\
6 \\
8\end{array}$ & $\begin{array}{l}\mathbf{5 , 2 5} \\
\mathbf{5 , 2 7} \\
\mathbf{5 , 3 0} \\
\mathbf{5 , 3 3} \\
\mathbf{5 , 3 5}\end{array}$ & \begin{tabular}{r|}
420 \\
2 \\
4 \\
6 \\
8
\end{tabular} & $\begin{array}{l}5,38 \\
5,40 \\
5,43 \\
5,45 \\
\mathbf{5 , 4 8}\end{array}$ & $\begin{array}{r}0430 \\
2 \\
4 \\
6 \\
8\end{array}$ & $\begin{array}{l}5,50 \\
5,53 \\
5,56 \\
5,58 \\
5,61\end{array}$ & $\begin{array}{r}r 40 \\
2 \\
4 \\
6 \\
8\end{array}$ & $\begin{array}{l}\mathbf{5 , 6 3} \\
\mathbf{5 , 6 6} \\
\mathbf{5 , 6 8} \\
\mathbf{5}, \mathbf{7 1} \\
\mathbf{5 , 7 3}\end{array}$ & $\begin{array}{r}r 40 \\
2 \\
4 \\
6 \\
8\end{array}$ & $\begin{array}{l}5,76 \\
5,79 \\
5,81 \\
5,84 \\
5,86\end{array}$ & $\begin{array}{r}0460 \\
2 \\
4 \\
6 \\
8\end{array}$ & $\begin{array}{l}5,89 \\
5,91 \\
5,94 \\
5,97 \\
5,99\end{array}$ & $\begin{array}{r}0470 \\
2 \\
4 \\
6 \\
8\end{array}$ & $\begin{array}{l}6,02 \\
6,04 \\
6.07 \\
6,09 \\
6,12\end{array}$ & $\begin{array}{r}480 \\
2 \\
4 \\
6 \\
8\end{array}$ & $\begin{array}{l}\mathbf{6 , 1 4} \\
6,17 \\
6,20 \\
6,22 \\
6,25\end{array}$ & $\begin{array}{r}0490 \\
2 \\
4 \\
6 \\
8\end{array}$ & $\begin{array}{l}6,27 \\
6,30 \\
6,32 \\
6,35 \\
6,37\end{array}$ \\
\hline $\begin{array}{r}500 \\
2 \\
4 \\
6 \\
8\end{array}$ & $\begin{array}{l}6.40 \\
6,43 \\
6,45 \\
6,48 \\
6,50\end{array}$ & $\begin{array}{r}510 \\
2 \\
4 \\
6 \\
8\end{array}$ & $\begin{array}{l}6,53 \\
6,55 \\
6,58 \\
6,61 \\
6,63\end{array}$ & $\begin{array}{r}520 \\
2 \\
4 \\
6 \\
8\end{array}$ & $\begin{array}{l}6.66 \\
6,18 \\
6,71 \\
6,73 \\
6,76\end{array}$ & $\begin{array}{r}8530 \\
2 \\
4 \\
6 \\
8\end{array}$ & $\begin{array}{l}6,78 \\
6,81 \\
6,84 \\
6,86 \\
6.89\end{array}$ & $\begin{array}{r}0540 \\
2 \\
4\end{array}$ & $\begin{array}{l}6,91 \\
6,94 \\
6,96 \\
6,99 \\
7,01\end{array}$ & $\begin{array}{r}0,0550 \\
2 \\
4\end{array}$ & $\begin{array}{l}7,04 \\
7,07 \\
\mathbf{7 , 0 9} \\
\mathbf{7 , 1 2} \\
\mathbf{7 , 1 4}\end{array}$ & $\begin{array}{r}0,0560 \\
2 \\
4 \\
6 \\
8\end{array}$ & $\begin{array}{l}7,17 \\
7,19 \\
7,22 \\
7,25 \\
7,27\end{array}$ & $\begin{array}{r}0570 \\
2 \\
4 \\
6 \\
8\end{array}$ & $\begin{array}{l}7,30 \\
7,32 \\
7,35 \\
7,37 \\
\mathbf{7 , 4 0}\end{array}$ & $\begin{array}{r}0,0580 \\
2 \\
4 \\
6\end{array}$ & $\begin{array}{l}7,42 \\
7,45 \\
7,48 \\
7,50 \\
7,53\end{array}$ & $\begin{array}{r}0,0590 \\
2 \\
4 \\
6\end{array}$ & $\begin{array}{l}7,55 \\
7,55 \\
7,60 \\
7,63 \\
7,65\end{array}$ \\
\hline $\begin{array}{r}600 \\
2 \\
4 \\
6 \\
8\end{array}$ & $\begin{array}{l}7,68 \\
7,71 \\
7,73 \\
7,76 \\
7,78\end{array}$ & $\begin{array}{r}610 \\
2 \\
4 \\
6 \\
8\end{array}$ & $\begin{array}{l}7,81 \\
7,83 \\
7,86 \\
7,89 \\
7,91\end{array}$ & $\begin{array}{r}620 \\
2 \\
4 \\
6 \\
8\end{array}$ & $\begin{array}{l}7,94 \\
7,96 \\
7,99 \\
8,01 \\
8,04\end{array}$ & $\begin{array}{r}630 \\
2 \\
4\end{array}$ & $\begin{array}{l}8,06 \\
8,09 \\
8,12 \\
8,14 \\
8,17\end{array}$ & $\begin{array}{r}0640 \\
2 \\
4\end{array}$ & $\begin{array}{l}8,19 \\
8,22\end{array}$ & $\left|\begin{array}{r}0,0650 \\
2\end{array}\right|$ &, 32 & $\begin{array}{r}9660 \\
2 \\
4 \\
6 \\
8\end{array}$ & $\begin{array}{l}8,45 \\
8,47 \\
8,50\end{array}$ & \begin{tabular}{|r|}
0,0670 \\
$\mathbf{2}$ \\
$\mathbf{4}$ \\
$\mathbf{6}$ \\
8
\end{tabular} & $\begin{array}{l}8,58 \\
860\end{array}$ & $\left|\begin{array}{r}0,0680 \\
2 \\
4 \\
6 \\
8\end{array}\right|$ & $\begin{array}{l}8,70 \\
8,73 \\
8,76 \\
8,78 \\
8,81\end{array}$ & $\begin{array}{r}0,0690 \\
\mathbf{2} \\
4 \\
6 \\
8\end{array}$ & $\begin{array}{l}8,83 \\
8,86 \\
8,88 \\
8,91 \\
8,93\end{array}$ \\
\hline $\begin{array}{r}700 \\
2 \\
4 \\
6 \\
8\end{array}$ & & $\begin{array}{r}710 \\
2 \\
4 \\
6 \\
8\end{array}$ & $\begin{array}{l}9,09 \\
\mathbf{9}, 11 \\
\mathbf{9 , 1 4} \\
\mathbf{9 , 1 7} \\
\mathbf{9 , 1 9}\end{array}$ & $\begin{array}{r}720 \\
2 \\
4 \\
6 \\
8\end{array}$ & $\begin{array}{l}9,22 \\
9,24 \\
9,27 \\
9,29 \\
9,32\end{array}$ & $\begin{array}{r}730 \\
2 \\
4 \\
6 \\
8\end{array}$ & $\begin{array}{l}9,34 \\
9,37 \\
9,40\end{array}$ & $\left|\begin{array}{r}0,0740 \\
2 \\
4\end{array}\right|$ & $\begin{array}{l}\mathbf{9 , 4 7} \\
\mathbf{9 , 5 0}\end{array}$ & $\begin{array}{r}0,0750 \\
2 \\
4\end{array}$ & $\begin{array}{l}9,60 \\
9,63\end{array}$ & $\begin{array}{r}0760 \\
2 \\
4 \\
6 \\
8\end{array}$ & $\begin{array}{l}9,73 \\
9,75\end{array}$ & $\begin{array}{r}0770 \\
2 \\
4 \\
6 \\
8\end{array}$ & & $\begin{array}{r}0,0780 \\
2 \\
4 \\
6 \\
8\end{array}$ & & $\begin{array}{r}0,0790 \\
2 \\
4 \\
6 \\
8\end{array}$ & $\begin{array}{l}10,11 \\
10,14 \\
10,16 \\
10,19 \\
10,21\end{array}$ \\
\hline $\begin{array}{r}800 \\
2 \\
4 \\
6 \\
8\end{array}$ & $\begin{array}{l}, 24 \\
, 27 \\
, 29 \\
, 32 \\
, 34\end{array}$ & $\begin{array}{r}810 \\
2 \\
4 \\
6 \\
8\end{array}$ & & $\begin{array}{r}820 \\
2 \\
4 \\
6 \\
8\end{array}$ & & $\begin{array}{r}830 \\
2 \\
4 \\
6 \\
8\end{array}$ & & $\begin{array}{r}840 \\
2 \\
4 \\
6 \\
8\end{array}$ & & $\begin{array}{r}850 \\
2 \\
4 \\
6\end{array}$ & & $\begin{array}{r}0860 \\
2 \\
4 \\
6 \\
8\end{array}$ & & $\begin{array}{r}0870 \\
2 \\
4 \\
6 \\
8\end{array}$ & & $\begin{array}{r}0,0880 \\
2 \\
\mathbf{4} \\
6 \\
8\end{array}$ & & $\begin{array}{r}90 \\
2 \\
4 \\
6 \\
8\end{array}$ & $\begin{array}{l}11,42 \\
11,44 \\
11,47 \\
11,49\end{array}$ \\
\hline $\begin{array}{r}1900 \\
2 \\
4 \\
6 \\
8\end{array}$ & 11,62 & $\begin{array}{r}910 \\
2 \\
4 \\
6 \\
8\end{array}$ & & $\begin{array}{r}920 \\
2 \\
4 \\
6 \\
8\end{array}$ & & $\begin{array}{r}930 \\
2 \\
4 \\
6 \\
8\end{array}$ & & $\begin{array}{r}940 \\
2 \\
4 \\
6 \\
8\end{array}$ & & $\begin{array}{r}950 \\
2 \\
4 \\
6 \\
8\end{array}$ & & $\begin{array}{r}60 \\
2 \\
4 \\
6 \\
8\end{array}$ & & \begin{tabular}{r|}
0970 \\
2 \\
4 \\
6 \\
8
\end{tabular} & & $\begin{array}{r}80 \\
2 \\
4 \\
6 \\
8\end{array}$ & & $\begin{array}{r}90 \\
2 \\
4 \\
6\end{array}$ & $\begin{array}{l}12,75 \\
12,77\end{array}$ \\
\hline $\begin{array}{r}000 \\
2 \\
4 \\
6 \\
8\end{array}$ & $\begin{array}{l}1 \\
1 \\
1 \\
1 \\
1\end{array}$ & $\begin{array}{r}010 \\
2 \\
4 \\
6 \\
8\end{array}$ & 3 & $\begin{array}{r}.020 \\
2 \\
4 \\
6 \\
8\end{array}$ & & $\begin{array}{r}1030 \\
2 \\
4 \\
6 \\
8\end{array}$ & \begin{tabular}{|l|}
13,18 \\
13,21 \\
13,24 \\
13,26 \\
13,29
\end{tabular} & $\begin{array}{r}1040 \\
2 \\
4 \\
6 \\
8\end{array}$ & 13,41 & $\begin{array}{r}.050 \\
2 \\
4\end{array}$ & & $\begin{array}{r}60 \\
2 \\
4 \\
6 \\
8\end{array}$ & & $\begin{array}{r}70 \\
2 \\
4 \\
6 \\
8\end{array}$ & & $\begin{array}{r}80 \\
2 \\
4 \\
6 \\
8\end{array}$ & & $\begin{array}{r}90 \\
2\end{array}$ & 14,05 \\
\hline $\begin{array}{r}100 \\
2 \\
\mathbf{4} \\
\mathbf{6} \\
8\end{array}$ & $\begin{array}{l}8 \\
6 \\
8\end{array}$ & $\begin{array}{r}110 \\
2 \\
4 \\
6 \\
8\end{array}$ & $\begin{array}{l}14,23 \\
14,26 \\
14,29 \\
14,31\end{array}$ & $\begin{array}{r}20 \\
2 \\
4 \\
6 \\
8\end{array}$ & & $\begin{array}{r}30 \\
2 \\
4 \\
6 \\
8\end{array}$ & & \begin{tabular}{r|r}
140 \\
2 \\
4 \\
6 \\
8
\end{tabular} & & $\begin{array}{r}50 \\
2 \\
4\end{array}$ & & $\begin{array}{r}60 \\
2 \\
4 \\
6 \\
8\end{array}$ & & $\begin{array}{r}70 \\
2 \\
4 \\
6 \\
8\end{array}$ & & $\begin{array}{r}80 \\
2 \\
4 \\
6 \\
8\end{array}$ & & $\begin{array}{r}90 \\
2\end{array}$ & $\begin{array}{l}15,20 \\
15,28 \\
15,31 \\
15,33\end{array}$ \\
\hline $\begin{array}{r}200 \\
2 \\
4 \\
6 \\
8\end{array}$ & 6,46 & $\begin{array}{r}10 \\
2 \\
4 \\
6 \\
8\end{array}$ & 15,59 & $\begin{array}{r}220 \\
2 \\
4 \\
6 \\
8\end{array}$ & $\begin{array}{l}15,64 \\
15,67 \\
15,69 \\
15,72\end{array}$ & $\begin{array}{r}30 \\
2 \\
4 \\
6 \\
8\end{array}$ & $\mid \begin{array}{l}15,80 \\
15,82 \\
15,85\end{array}$ & $\begin{array}{r}40 \\
2 \\
4 \\
6 \\
8\end{array}$ & \begin{tabular}{|l|}
15,95 \\
15,97
\end{tabular} \mid & $\begin{array}{r}50 \\
2 \\
4 \\
6 \\
8\end{array}$ & 16,10 & $\begin{array}{r}60 \\
2 \\
4 \\
6 \\
8\end{array}$ & $\begin{array}{l}16,21 \\
16,23\end{array}$ & $\begin{array}{r}70 \\
2 \\
4 \\
6 \\
8\end{array}$ & 16,36 & $\begin{array}{r}80 \\
2 \\
4 \\
6 \\
8\end{array}$ & 16,49 & $\begin{array}{r}290 \\
\mathbf{2}\end{array}$ & $\begin{array}{l}16,59 \\
16,61\end{array}$ \\
\hline
\end{tabular}

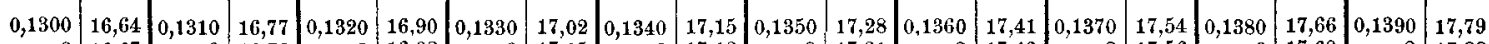

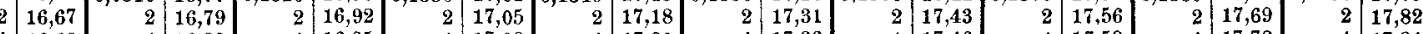

\begin{tabular}{l|l|l|l|l|l|l|l|l|l|l|l|l|l|r|r|r|r|r|r|}
4 & 16,69 & 4 & 16,82 & 4 & 16,95 & 4 & 17,08 & 4 & 17,20 & 4 & 17,33 & 4 & 17,46 & 4 & 17,59 & 4 & 17,72 & 4 & 17,84
\end{tabular}

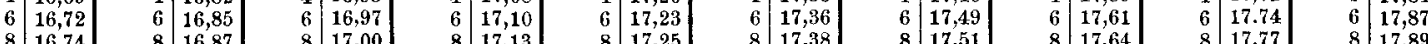

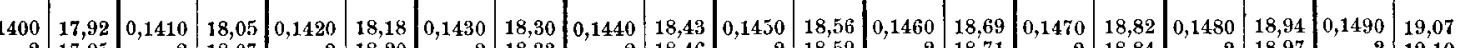

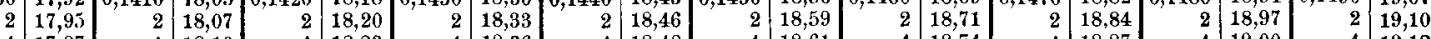

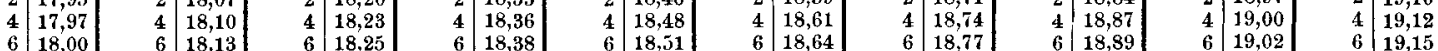

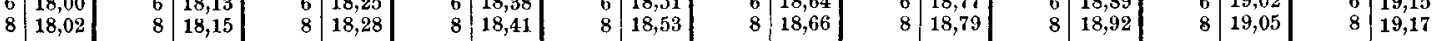

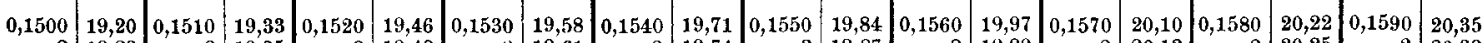

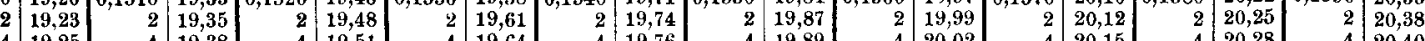

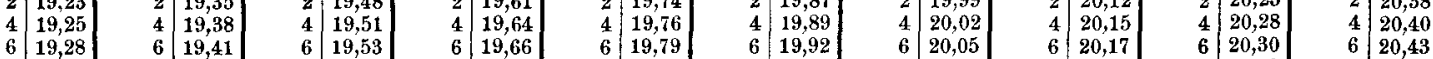

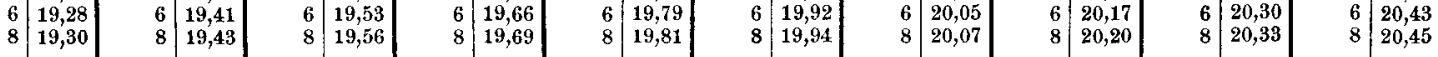

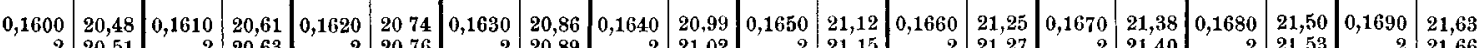

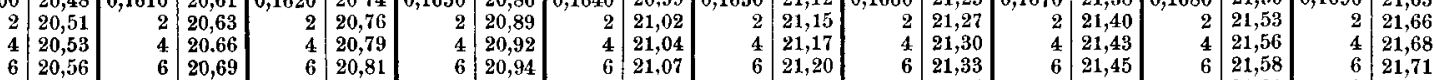

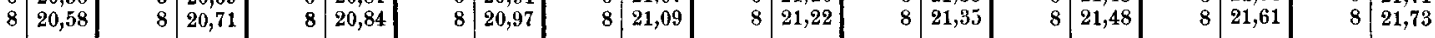

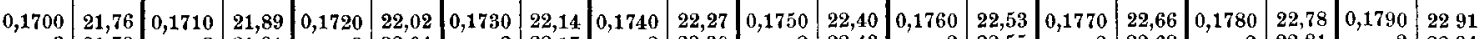

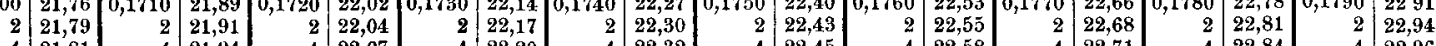

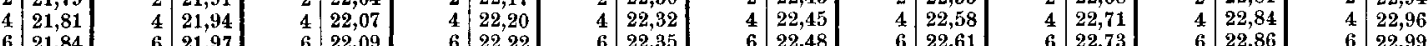

\begin{tabular}{l|l|l|l|l|l|l|l|l|l|l|l|l|r|r|r|r|r|r|r|}
6 & 21,84 & 6 & 21,97 & 6 & 22,09 & 6 & 22,22 & 6 & 22,35 & 6 & 22,48 & 6 & 22,61 & 6 & 22,73 & 6 & 22,86 & 6 & 22,99 \\
8 & 21,86 & 8 & 21,99 & 8 & 22,12 & 8 & 22,25 & 8 & 22,37 & 8 & 22,50 & 8 & 22,63 & 8 & 22,76 & 8 & 22,89 & 8 & 23,01
\end{tabular}

\begin{tabular}{r|r|r|r|r|r|r|r|r|r|r|r|r|r|r|r|r|r|r|r|r|r|}
0,1800 & 23,04 & 0,1810 & 23,17 & 0,1820 & 23,30 & 0,1830 & 23,42 & 0,1840 & 23,55 & 0,1850 & 23,68 & 0,1860 & 23,81 & 0,1870 & 23,94 & 0,1880 & 24,06 & 0,1890 & 24,19
\end{tabular}

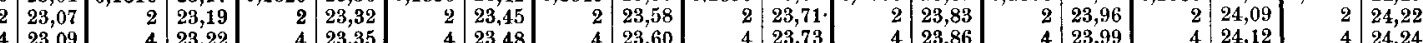

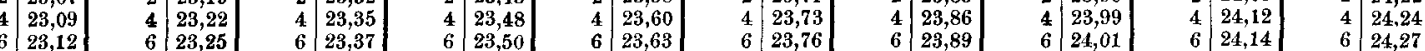

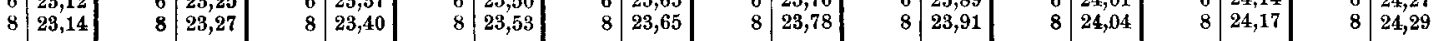

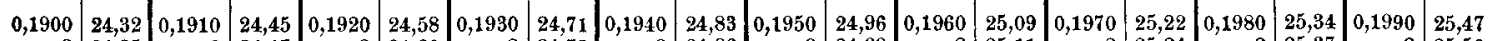

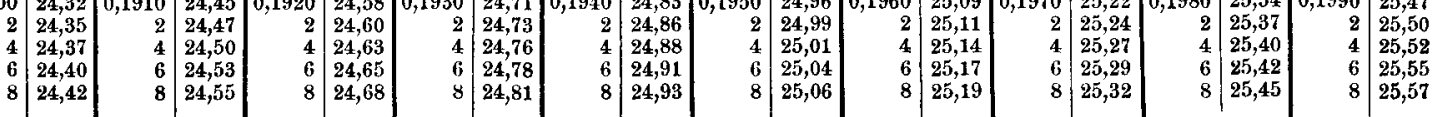




\begin{tabular}{|c|c|c|c|c|c|c|c|c|c|c|c|c|c|c|c|c|c|c|c|}
\hline $\begin{array}{l}0 \\
2 \\
\infty \\
\infty \\
\infty\end{array}$ & $\begin{array}{l}0 \\
0 \\
0 \\
0 \\
0 \\
0\end{array}$ & 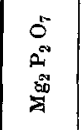 & $\begin{array}{l}0 \\
0 \\
01\end{array}$ & $\begin{array}{l}0 \\
0 \\
0 \\
\infty \\
\Sigma\end{array}$ & $\begin{array}{l}0 \\
0 \\
01\end{array}$ & $\begin{array}{l}0 \\
\infty^{\infty} \\
\sum^{\infty}\end{array}$ & $\begin{array}{l}0 \\
0 \\
2\end{array}$ & $\begin{array}{l}0^{\infty} \\
2^{\infty} \\
\underbrace{5}\end{array}$ & $\begin{array}{l}0 \\
0\end{array}$ & $\begin{array}{l}0 \\
0 \\
0 \\
0 \\
0 \\
0\end{array}$ & $0_{0}^{\infty}$ & $\begin{array}{l}\hat{0} \\
0=1 \\
0 \\
0\end{array}$ & $0_{\infty}^{\infty}$ & $\begin{array}{l}0 \\
0 \\
0 \\
0\end{array}$ & $\begin{array}{l}0 \\
2\end{array}$ & $\begin{array}{l}5 \\
0 \\
0 \\
0\end{array}$ & $\oint_{0}^{6}$ & $\begin{array}{l}5 \\
5 \\
0^{\circ} \\
\sum^{\infty}\end{array}$ & $\begin{array}{l}0 \\
0 \\
0\end{array}$ \\
\hline $\begin{array}{r}0,2000 \\
2 \\
4 \\
6 \\
8\end{array}$ & $\begin{array}{l}25,60 \\
25,63 \\
25,65 \\
25,68 \\
25,70\end{array}$ & $\mid$\begin{tabular}{r|}
0,2010 \\
2 \\
4 \\
6 \\
8
\end{tabular} & $\begin{array}{l}25,73 \\
25,75 \\
25,78 \\
45,81 \\
25,83\end{array}$ & $\begin{array}{r}0,2020 \\
2 \\
4 \\
6 \\
8\end{array}$ & $\begin{array}{l}25,86 \\
25,88 \\
25,91 \\
25,93 \\
25,96\end{array}$ & $\begin{array}{r}0.2030 \\
2 \\
4 \\
6 \\
8\end{array}$ & $\begin{array}{l}25,98 \\
26,01 \\
26,04 \\
\mathbf{2 6}, 06 \\
\mathbf{2 6 , 0 9}\end{array}$ & $\begin{array}{r}0,2040 \\
2 \\
4 \\
6 \\
8\end{array}$ & $\begin{array}{l}26,11 \\
26,14 \\
26,16 \\
26,19 \\
26,21\end{array}$ & $\begin{array}{r}0,2050 \\
2 \\
4 \\
6 \\
8\end{array}$ & $\begin{array}{l}26,24 \\
26,27 \\
26,29 \\
26,39 \\
\mathbf{2 6 , 3 4}\end{array}$ & $\begin{array}{r}0,2060 \\
2 \\
4 \\
6 \\
8\end{array}$ & $\begin{array}{l}26,37 \\
26,39 \\
26,42 \\
26,45 \\
26,47\end{array}$ & $\begin{array}{r}0,2070 \\
2 \\
4 \\
6 \\
8\end{array}$ & $\begin{array}{l}26,50 \\
26,52 \\
26,55 \\
26,57 \\
26,60\end{array}$ & $\begin{array}{r}0,2080 \\
2 \\
4 \\
6 \\
8\end{array}$ & $\begin{array}{l}26,62 \\
26,65 \\
26,68 \\
26,70 \\
26,73\end{array}$ & $\mid \begin{array}{r}0,2090 \\
2 \\
4 \\
6 \\
8\end{array}$ & $\begin{array}{l}26,75 \\
26,78 \\
26,80 \\
26,83 \\
26,85\end{array}$ \\
\hline $\begin{array}{r}0,2100 \\
2 \\
4 \\
6 \\
8\end{array}$ & $\begin{array}{l}26,88 \\
26,91 \\
26,93 \\
26,96 \\
26,98\end{array}$ & $\begin{array}{r}0,2110 \\
2 \\
4 \\
6 \\
8\end{array}$ & $\begin{array}{l}27,01 \\
27,03 \\
27,06 \\
27,09 \\
27,11\end{array}$ & $\begin{array}{r}0,2120 \\
2 \\
4 \\
6 \\
8\end{array}$ & $\begin{array}{l}27,14 \\
27,16 \\
27,19 \\
27,21 \\
27,24\end{array}$ & $\begin{array}{r}0,2130 \\
2 \\
4 \\
6 \\
8\end{array}$ & $\begin{array}{l}27,26 \\
27,29 \\
27,32 \\
27,34 \\
27,37\end{array}$ & $\begin{array}{r}0,2140 \\
2 \\
4 \\
6 \\
8\end{array}$ & $\begin{array}{l}27,39 \\
27,42 \\
27,44 \\
27,47 \\
27,49\end{array}$ & $\begin{array}{r}0,2150 \\
2 \\
4 \\
6 \\
8\end{array}$ & $\begin{array}{l}27,52 \\
27,55 \\
27,57 \\
27,60 \\
27,62\end{array}$ & $\begin{array}{r}0,2160 \\
2 \\
4 \\
6 \\
8\end{array}$ & $\begin{array}{l}2765 \\
27,67 \\
27,70 \\
27,73 \\
27,75\end{array}$ & $\begin{array}{r}0,2170 \\
2 \\
4 \\
6 \\
8\end{array}$ & $\begin{array}{l}27,78 \\
27,80 \\
27,83 \\
27,85 \\
27,88\end{array}$ & $\begin{array}{r}0,2180 \\
2 \\
4 \\
6 \\
8\end{array}$ & $\begin{array}{l}27,90 \\
27,93 \\
27,96 \\
27,98 \\
28,01\end{array}$ & $\mid \begin{array}{r}0,2190 \\
2 \\
4 \\
6 \\
8\end{array}$ & $\begin{array}{l}28,03 \\
28,06 \\
28,08 \\
28,11 \\
28,13\end{array}$ \\
\hline $\begin{array}{r}0,2200 \\
2 \\
4 \\
6 \\
8\end{array}$ & $\begin{array}{l}28,16 \\
28,19 \\
28,21 \\
28,24 \\
28,26\end{array}$ & \begin{tabular}{|r|}
0,2210 \\
2 \\
4 \\
6 \\
8
\end{tabular} & $\begin{array}{l}28,29 \\
28,31 \\
28,34 \\
28,37 \\
28,39\end{array}$ & $\begin{array}{r}0,2220 \\
2 \\
4 \\
6 \\
8\end{array}$ & $\begin{array}{l}28,42 \\
28,44 \\
28,47 \\
28,49 \\
28,52\end{array}$ & $\mid \begin{array}{r}0,2230 \\
2 \\
4 \\
6 \\
8\end{array}$ & $\begin{array}{l}28,54 \\
28,57 \\
28,60 \\
28,62 \\
28,65\end{array}$ & $\begin{array}{r}0,2240 \\
2 \\
4 \\
6 \\
8\end{array}$ & $\begin{array}{l}28,67 \\
28,70 \\
28,72 \\
28,75 \\
28,77\end{array}$ & $\begin{array}{r}0,2250 \\
2 \\
4 \\
6 \\
8\end{array}$ & $\begin{array}{l}28,80 \\
28,83 \\
28,85 \\
28,88 \\
28,90\end{array}$ & $\begin{array}{r}0,2260 \\
2 \\
4 \\
6 \\
8\end{array}$ & $\begin{array}{l}28,93 \\
28,95 \\
28,98 \\
29,01 \\
29,03\end{array}$ & $\mid \begin{array}{r}0,2270 \\
2 \\
4 \\
6 \\
8\end{array}$ & $\begin{array}{l}29,06 \\
29,08 \\
29,11 \\
29,13 \\
29,16\end{array}$ & $\begin{array}{r}0,2280 \\
2 \\
4 \\
6 \\
8\end{array}$ & $\begin{array}{l}29,18 \\
29,21 \\
29,24 \\
29,26 \\
29,29\end{array}$ & $\mid \begin{array}{r}0,2290 \\
2 \\
4 \\
6 \\
8\end{array}$ & $\begin{array}{l}29,31 \\
29,34 \\
29,36 \\
29,39 \\
29,41\end{array}$ \\
\hline $\begin{array}{r}0,2300 \\
2 \\
4 \\
6 \\
8\end{array}$ & $\begin{array}{l}29,44 \\
29,47 \\
29, \mathbf{4 9} \\
29,52 \\
\mathbf{2 9 , 5 4}\end{array}$ & $\left|\begin{array}{r}0,2310 \\
2 \\
. \quad 4 \\
6 \\
8\end{array}\right|$ & $\begin{array}{l}29,57 \\
29,59 \\
29,62 \\
29,65 \\
29,67\end{array}$ & $\begin{array}{r}0,2320 \\
2 \\
4 \\
6 \\
8\end{array}$ & $\begin{array}{l}29,70 \\
29,72 \\
29,75 \\
29,77 \\
29,80\end{array}$ & $\begin{array}{r}0,2330 \\
2 \\
4 \\
6 \\
8\end{array}$ & $\begin{array}{l}29,82 \\
29,85 \\
29,88 \\
29,90 \\
29,93\end{array}$ & $\begin{array}{r}0,2340 \\
2 \\
4 \\
6 \\
8\end{array}$ & $\begin{array}{l}29,95 \\
29,98 \\
30,00 \\
30,03 \\
30,05\end{array}$ & \begin{tabular}{|r|}
0,2350 \\
2 \\
4 \\
6 \\
8
\end{tabular} & $\begin{array}{l}30,08 \\
30,11 \\
30,13 \\
30,16 \\
30,18\end{array}$ & $\begin{array}{r}0,2360 \\
\mathbf{2} \\
4 \\
6 \\
8\end{array}$ & $\begin{array}{l}30,21 \\
30,23 \\
30,26 \\
30,29 \\
30,31\end{array}$ & $\begin{array}{r}0,2370 \\
2 \\
4 \\
6 \\
8\end{array}$ & \begin{tabular}{|l|}
30.34 \\
30,36 \\
30.39 \\
30,41 \\
30.44
\end{tabular} & $\begin{array}{r}0,2380 \\
2 \\
4 \\
6 \\
8\end{array}$ & $\begin{array}{l}30,46 \\
30.49 \\
30,52 \\
30.54 \\
30,57\end{array}$ & $\begin{array}{r}0,2390 \\
2 \\
4 \\
6 \\
8\end{array}$ & $\begin{array}{l}30,59 \\
30,62 \\
30,64 \\
30,67 \\
30,69\end{array}$ \\
\hline $\begin{array}{r}0,2400 \\
2 \\
4 \\
6 \\
8\end{array}$ & $\begin{array}{l}30,72 \\
30,75 \\
30,77 \\
30,80 \\
30,82\end{array}$ & $\left|\begin{array}{r}0,2410 \\
2 \\
4 \\
6 \\
8\end{array}\right|$ & $\begin{array}{l}30,85 \\
30,87 \\
30,9) \\
30,93 \\
30,95\end{array}$ & $\begin{array}{r}0,2420 \\
2 \\
4 \\
6 \\
8\end{array}$ & $\begin{array}{l}30,98 \\
31,00 \\
31.03 \\
31,05 \\
31,08\end{array}$ & $\mid \begin{array}{r}0,2430 \\
2 \\
4 \\
6 \\
8\end{array}$ & $\begin{array}{l}31,10 \\
31,13 \\
31,16 \\
31,18 \\
31,21\end{array}$ & $\begin{array}{r}0,2440 \\
2 \\
4 \\
6 \\
8\end{array}$ & $\begin{array}{l}\mathbf{3 1}, \mathbf{2 3} \\
\mathbf{3 1}, 26 \\
\mathbf{3 1}, 28 \\
\mathbf{3 1}, \mathbf{3 1} \\
\mathbf{3 1}, \mathbf{3 3}\end{array}$ & $\mid \begin{array}{r}0,2450 \\
2 \\
4 \\
6 \\
8\end{array}$ & $\begin{array}{l}31,36 \\
31,39 \\
31,41 \\
31,44 \\
31,46\end{array}$ & $\begin{array}{r}0,2460 \\
\mathbf{2} \\
4 \\
\mathbf{6} \\
8\end{array}$ & $\begin{array}{l}31,49 \\
31,51 \\
31,54 \\
31,57 \\
31,59\end{array}$ & $\begin{array}{r}0,2470 \\
2 \\
4 \\
6 \\
8\end{array}$ & $\begin{array}{l}31,62 \\
31,64 \\
31,67 \\
31,69 \\
31,72\end{array}$ & $\begin{array}{r}0,2480 \\
2 \\
4 \\
6 \\
8\end{array}$ & $\begin{array}{l}31,74 \\
31,77 \\
31,80 \\
31,82 \\
31,85\end{array}$ & $\mid \begin{array}{r}0,2490 \\
2 \\
4 \\
6 \\
8\end{array}$ & $\begin{array}{l}31,87 \\
31,90 \\
31,92 \\
31,95 \\
31,97\end{array}$ \\
\hline $\begin{array}{r}0,2500 \\
2 \\
4 \\
6 \\
8\end{array}$ & $\begin{array}{l}32,00 \\
32,03 \\
32,05 \\
32,08 \\
32,10\end{array}$ & $\begin{array}{r}0,2510 \\
2 \\
4 \\
6 \\
8\end{array}$ & $\begin{array}{l}32,13 \\
32,15 \\
32,17 \\
32,21 \\
32,23\end{array}$ & $\begin{array}{r}0,2520 \\
2 \\
4 \\
6 \\
8\end{array}$ & $\begin{array}{l}32,26 \\
32,28 \\
32,31 \\
32,33 \\
32,36\end{array}$ & $\begin{array}{r}0,2530 \\
2 \\
4 \\
6 \\
8\end{array}$ & $\begin{array}{l}32,38 \\
32,41 \\
32,44 \\
32,46 \\
32,49\end{array}$ & $\begin{array}{r}0.2540 \\
2 \\
4 \\
6 \\
8\end{array}$ & $\begin{array}{l}32,51 \\
32,54 \\
32,56 \\
32,59 \\
32,61\end{array}$ & $\mid \begin{array}{r}0,2550 \\
2 \\
4 \\
6 \\
8\end{array}$ & $\begin{array}{l}32,64 \\
32,67 \\
32,69 \\
32,72 \\
32,74\end{array}$ & $\begin{array}{r}0,2560 \\
2 \\
4 \\
6 \\
8\end{array}$ & $\begin{array}{l}32,77 \\
32,79 \\
32,82 \\
32,85 \\
32,87\end{array}$ & $\left|\begin{array}{r}0,2570 \\
2 \\
4 \\
6 \\
8\end{array}\right|$ & $\begin{array}{l}32,90 \\
32,92 \\
32,95 \\
32,97 \\
33,00\end{array}$ & $\mid \begin{array}{r}0,2580 \\
2 \\
4 \\
6 \\
8\end{array}$ & $\begin{array}{l}33,02 \\
33,05 \\
33,08 \\
33,10 \\
33,13\end{array}$ & $\mid \begin{array}{r}0,2590 \\
2 \\
4 \\
6 \\
8\end{array}$ & $\begin{array}{l}33,15 \\
33,18 \\
33,20 \\
33,23 \\
33,25\end{array}$ \\
\hline $\begin{array}{r}0,2600 \\
2 \\
4 \\
6 \\
8\end{array}$ & $\begin{array}{l}33,28 \\
33,31 \\
33,33 \\
33,36 \\
33,38\end{array}$ & $\begin{array}{r}0,2610 \\
2 \\
4 \\
6 \\
8\end{array}$ & $\begin{array}{l}33,41 \\
33,43 \\
33,46 \\
33,49 \\
33,51\end{array}$ & $\begin{array}{r}0,2620 \\
z \\
4 \\
6 \\
8\end{array}$ & $\begin{array}{l}33,54 \\
33,56 \\
33,59 \\
33,61 \\
33,64\end{array}$ & \begin{tabular}{rr|}
02630 \\
2 \\
4 \\
\\
\\
\\
\\
4 \\
8
\end{tabular} & $\begin{array}{l}33,66 \\
33,69 \\
33,72 \\
33,74 \\
33,77\end{array}$ & $\begin{array}{r}0,2640 \\
2 \\
4 \\
6 \\
8\end{array}$ & $\begin{array}{l}33,79 \\
33,82 \\
33,84 \\
33,87 \\
33,89\end{array}$ & $\begin{array}{r}0.2650 \\
2 \\
4 \\
6 \\
8\end{array}$ & $\begin{array}{l}33,92 \\
33,95 \\
33,97 \\
34,00 \\
34,02\end{array}$ & $\begin{array}{r}0,2660 \\
2 \\
4 \\
6 \\
8\end{array}$ & $\begin{array}{l}34,05 \\
34,07 \\
34,10 \\
34,13 \\
34,15\end{array}$ & $\begin{array}{r}0,2670 \\
2 \\
4 \\
6 \\
8\end{array}$ & $\begin{array}{l}34,18 \\
34,20 \\
34,23 \\
34,25 \\
34,25\end{array}$ & $\begin{array}{r}0,2680 \\
2 \\
4 \\
6 \\
8\end{array}$ & $\begin{array}{l}34,30 \\
34,33 \\
34,36 \\
34,38 \\
34,41\end{array}$ & $\mid \begin{array}{r}0,2690 \\
2 \\
4 \\
6 \\
8\end{array}$ & $\begin{array}{l}34,43 \\
34,46 \\
34,48 \\
34,51 \\
34,53\end{array}$ \\
\hline $\begin{array}{r}2700 \\
2 \\
4 \\
6 \\
8\end{array}$ & $\begin{array}{l}34,56 \\
34,59 \\
34,61 \\
34,64 \\
34,66\end{array}$ & $\begin{array}{r}0,2710 \\
2 \\
4 \\
6 \\
8\end{array}$ & $\begin{array}{l}34,69 \\
34,71 \\
34,74 \\
34,77 \\
34,79\end{array}$ & $\begin{array}{r}0,9720 \\
2 \\
4 \\
6 \\
8\end{array}$ & $\begin{array}{l}34,82 \\
34,84 \\
34.87 \\
34,89 \\
34,92\end{array}$ & $\begin{array}{r}0,2730 \\
2 \\
4 \\
6 \\
8\end{array}$ & $\begin{array}{l}34,94 \\
34,97 \\
35,00 \\
35,02 \\
35,05\end{array}$ & $\begin{array}{r}0,2740 \\
2 \\
4 \\
6 \\
8\end{array}$ & $\begin{array}{l}35,07 \\
35,10 \\
35,12 \\
35,15 \\
35,17\end{array}$ & $\begin{array}{r}0,2750 \\
2 \\
4 \\
6 \\
8\end{array}$ & $\begin{array}{l}35,20 \\
35,23 \\
35,25 \\
35,28 \\
35,30\end{array}$ & $\begin{array}{r}0,2760 \\
2 \\
4 \\
6 \\
8\end{array}$ & $\begin{array}{l}35,33 \\
35,35 \\
35,38 \\
35,41 \\
35,43\end{array}$ & $\left|\begin{array}{r}0,2770 \\
2 \\
4 \\
6 \\
8\end{array}\right|$ & $\begin{array}{l}35,46 \\
35,48 \\
35,51 \\
35,53 \\
35,56\end{array}$ & $\begin{array}{r}0,2780 \\
2 \\
4 \\
6 \\
8\end{array}$ & $\begin{array}{l}35,58 \\
35,61 \\
35,64 \\
35,66 \\
35,69\end{array}$ & $\begin{array}{r}0,2790 \\
2 \\
4 \\
6 \\
8\end{array}$ & $\begin{array}{l}35,71 \\
35,74 \\
35,76 \\
35,79 \\
35,81\end{array}$ \\
\hline $\begin{array}{r}2800 \\
2 \\
4 \\
6 \\
8\end{array}$ & $\begin{array}{l}\mathbf{3 5}, 84 \\
\mathbf{3 5}, 87 \\
\mathbf{3 5 , 8 9} \\
\mathbf{3 5 ,}, 92 \\
\mathbf{3 5 , 9 4}\end{array}$ & $\mid \begin{array}{r}0,2810 \\
2 \\
4 \\
6 \\
8\end{array}$ & $\begin{array}{l}35,97 \\
35,99 \\
36,02 \\
36,05 \\
36,07\end{array}$ & $\begin{array}{r}0,2820 \\
2 \\
4 \\
6 \\
8\end{array}$ & $\begin{array}{l}36,10 \\
36,12 \\
36,15 \\
36,17 \\
36,20\end{array}$ & $\begin{array}{r}0,2830 \\
2 \\
4 \\
6 \\
8\end{array}$ & $\begin{array}{l}36,22 \\
36,25 \\
36,28 \\
36,30 \\
36,33\end{array}$ & $\begin{array}{r}0,2840 \\
2 \\
4 \\
6 \\
8\end{array}$ & $\begin{array}{l}\mathbf{3 6 , 3 5} \\
36,38 \\
36,40 \\
36,43 \\
\mathbf{3 6 , 4 5}\end{array}$ & $\begin{array}{r}0.2850 \\
2 \\
4 \\
6 \\
8\end{array}$ & $\begin{array}{l}36,48 \\
36,51 \\
36,53 \\
36,56 \\
36,58\end{array}$ & $\begin{array}{r}0,2860 \\
2 \\
4 \\
6 \\
8\end{array}$ & $\begin{array}{l}36,61 \\
36,63 \\
36,66 \\
36,69 \\
36,71\end{array}$ & $\begin{array}{r}0,2870 \\
2 \\
4 \\
6 \\
8\end{array}$ & $\begin{array}{l}36,74 \\
36,76 \\
36,79 \\
36,81 \\
36,84\end{array}$ & $\begin{array}{r}0,2880 \\
2 \\
4 \\
6 \\
8\end{array}$ & $\begin{array}{l}36,86 \\
36,89 \\
36,92 \\
36,94 \\
36,97\end{array}$ & $\begin{array}{r}0,2890 \\
2 \\
4 \\
6 \\
8\end{array}$ & $\begin{array}{l}36,99 \\
37,02 \\
37,04 \\
37,07 \\
37,09\end{array}$ \\
\hline $\begin{array}{r}, 2900 \\
2 \\
4 \\
6 \\
8\end{array}$ & $\begin{array}{l}37,12 \\
37,15 \\
37,17 \\
37,20 \\
37,22\end{array}$ & $\mid \begin{array}{r}0,2910 \\
2 \\
4 \\
6 \\
8\end{array}$ & $\begin{array}{l}37,25 \\
37,27 \\
37,30 \\
37,33 \\
37,35\end{array}$ & $\begin{array}{r}0,9920 \\
2 \\
4 \\
6 \\
8\end{array}$ & $\begin{array}{l}37,38 \\
37,40 \\
37,43 \\
37,45 \\
37,48\end{array}$ & $\begin{array}{r}2930 \\
2 \\
4 \\
6 \\
8\end{array}$ & $\begin{array}{l}37,50 \\
37,53 \\
37,56 \\
37,58 \\
37,61\end{array}$ & $\begin{array}{r}0,2940 \\
2 \\
4 \\
6 \\
8\end{array}$ & $\begin{array}{l}37,63 \\
37,66 \\
37,68 \\
37,71 \\
37,73\end{array}$ & $\begin{array}{r}0,2950 \\
2 \\
4 \\
6 \\
8\end{array}$ & $\begin{array}{l}37,76 \\
37,79 \\
37,81 \\
37,84 \\
37,86\end{array}$ & $\begin{array}{r}0,2960 \\
2 \\
4 \\
6 \\
8\end{array}$ & $\begin{array}{l}37,89 \\
37,91 \\
37,94 \\
37,97 \\
37,99\end{array}$ & $\begin{array}{r}0,2970 \\
2 \\
4 \\
6 \\
8\end{array}$ & $\begin{array}{l}38,02 \\
38,04 \\
38,07 \\
38,09 \\
38,12\end{array}$ & $\begin{array}{r}0,2980 \\
2 \\
4 \\
6 \\
8\end{array}$ & $\begin{array}{l}38,14 \\
38,17 \\
38,20 \\
38,22 \\
38,25\end{array}$ & $\begin{array}{r}0,2990 \\
2 \\
4 \\
6 \\
8\end{array}$ & $\begin{array}{l}38,27 \\
38,30 \\
38,32 \\
38,35 \\
38,37\end{array}$ \\
\hline $\begin{array}{r}, 3000 \\
2 \\
4 \\
6 \\
8\end{array}$ & $\begin{array}{l}\mathbf{3 8 . 4 0} \\
38,43 \\
38,45 \\
38, \mathbf{4 8} \\
38,50\end{array}$ & $\begin{array}{r}0,3010 \\
2 \\
4 \\
6 \\
8\end{array}$ & $\begin{array}{l}38,53 \\
38,55 \\
38,58 \\
38,61 \\
38,63\end{array}$ & $\begin{array}{r}0,3020 \\
2 \\
4 \\
6 \\
8\end{array}$ & $\begin{array}{l}38,66 \\
38,68 \\
38,71 \\
38,73 \\
38,76\end{array}$ & $\begin{array}{r}, 3030 \\
2 \\
4 \\
6 \\
8\end{array}$ & $\begin{array}{l}38,78 \\
38,81 \\
38,84 \\
38,86 \\
38,89\end{array}$ & $\begin{array}{r}0,3040 \\
2 \\
4 \\
6 \\
8\end{array}$ & $\begin{array}{l}38,91 \\
38,94 \\
38,96 \\
38,99 \\
39,01\end{array}$ & $\begin{array}{r}0,3050 \\
2 \\
4 \\
6 \\
8\end{array}$ & $\begin{array}{l}39,04 \\
39,07 \\
39,09 \\
39,12 \\
39,14\end{array}$ & $\begin{array}{r}0,3060 \\
2 \\
4 \\
6 \\
8\end{array}$ & $\begin{array}{l}39,17 \\
39,19 \\
39,22 \\
39,25 \\
39,27\end{array}$ & $\begin{array}{r}0,3070 \\
2 \\
4 \\
6 \\
8\end{array}$ & $\begin{array}{l}39,30 \\
39,32 \\
39,35 \\
39,37 \\
39,40\end{array}$ & $\begin{array}{r}0,3080 \\
2 \\
4 \\
6 \\
8\end{array}$ & $\begin{array}{r}\mathbf{3 9 , 1 2} \\
\mathbf{3 9 , 4 5} \\
\mathbf{3 9 , 4 8} \\
39,50 \\
39,53\end{array}$ & $\begin{array}{r}0,3090 \\
2 \\
4 \\
6 \\
8\end{array}$ & $\begin{array}{l}39,55 \\
39,58 \\
39,60 \\
39,63 \\
39,65\end{array}$ \\
\hline $\begin{array}{r}100 \\
2 \\
4 \\
6 \\
8\end{array}$ & $\begin{array}{l}39,68 \\
39,71 \\
39,73 \\
39,76 \\
39,78\end{array}$ & $\begin{array}{r}3110 \\
2 \\
4 \\
6 \\
8\end{array}$ & $\begin{array}{l}39,81 \\
39,83 \\
39,86 \\
39,89 \\
39,91\end{array}$ & $\begin{array}{r}0,3120 \\
2 \\
4 \\
6 \\
8\end{array}$ & $\begin{array}{l}39,94 \\
39,96 \\
39,99 \\
40,01 \\
40,04\end{array}$ & $\begin{array}{r}3130 \\
2 \\
4 \\
6 \\
8\end{array}$ & $\begin{array}{l}40,06 \\
40.09 \\
40,12 \\
40,14 \\
40,17\end{array}$ & $\begin{array}{r}0,3140 \\
2 \\
4 \\
6 \\
8\end{array}$ & $\begin{array}{l}40,19 \\
40,22 \\
40,24 \\
40,27 \\
40,29\end{array}$ & $\begin{array}{r}3150 \\
2 \\
4 \\
6 \\
8\end{array}$ & $\begin{array}{l}40,39 \\
40,35 \\
40,37 \\
40,40 \\
40,42\end{array}$ & $\begin{array}{r}3160 \\
2 \\
4 \\
6 \\
8\end{array}$ & $\begin{array}{l}40,45 \\
40,47 \\
40,50 \\
40,53 \\
40,55\end{array}$ & $\begin{array}{r}3170 \\
2 \\
4 \\
6 \\
8\end{array}$ & \begin{tabular}{|l|}
40,58 \\
40,60 \\
40,63 \\
40,65 \\
40,68
\end{tabular} & $\begin{array}{r}0,3180 \\
2 \\
4 \\
6 \\
8\end{array}$ & $\begin{array}{l}40,70 \\
40,73 \\
40,76 \\
40,78 \\
40,81\end{array}$ & $\begin{array}{r}3190 \\
2 \\
4 \\
6 \\
8\end{array}$ & $\begin{array}{l}40,83 \\
40,86 \\
40,83 \\
40,91 \\
40,93\end{array}$ \\
\hline
\end{tabular}

Mittheilungen aus Schmitt's Laboratorium in Wiesbaden.

Studien aus dem Gebiete des bacteriologisch-bygienischen Untersuchungswesens.

\section{Von}

Val. Gerlach.

[Fortset:zung w. S. 72.j)

b) Über Safransurrogat. Nach Untersuchungen von Weyl (D. med. Wochenschr.
1886 S. 970, Ber. deutsch. G. 1887 S. 2835 ) ist das aus Dinitrokresolkalium bez. Ammonium bestehende Safransurrogat ein heftiges Gift. $\mathrm{Da}$ genannter Stoff durch $\mathrm{Be}^{-}$ schluss des Reichstages vom 15. Juni 1887 in die Reihe der erlaubten Farben aufgenommen wurde, erschien eine nähere Prüfung der Weyl'schen Bebauptung um so wichtiger, als in der Veröffentlichung Weyl's Vergiftungsversuche nur mit Kaninchen angestellt wurden. 